\title{
A spatial frequency effect on perceived depth
}

\author{
JAMES M. BROWN and NAOMI WEISSTEIN \\ State University of New York at Buffalo, Buffalo, New York
}

\begin{abstract}
We report a spatial frequency influence on perceptual organization. If regions filled with relatively higher spatial frequency sinusoidal gratings are adjacent to regions containing relatively lower spatial frequency gratings, the regions with the higher frequency will appear closer in depth than those containing the lower frequency. This depth effect is evident even when conflicting stereo information is present.
\end{abstract}

Previous work has revealed an intriguing phenomenon in apparent depth (Brown \& Weisstein, 1985). If alternate "bars" (horizontal rectangles) in a visual field are filled with sinusoidal gratings that differ in spatial frequency, the regions with the relatively higher frequency grating will appear closer in depth than the regions with the relatively lower frequency grating (Figure 1). This appearance of depth increases as the octave separation between the high- and low-frequency gratings increases. The effect is consistent, and, when considered in terms of relative size and detail perspective (Kaufman, 1974), it is also unexpected. According to these monocular depth cues, the larger bars of the lower frequency filled regions might be expected to provide depth/distance information indicating that they are nearer in depth than the narrower bars of the higher frequency filled regions.

There is increasing evidence of a link between the perception of depth and the sensitivity of the visual system to spatial frequency information. For example, Pentland (1985) has shown that the more an occluded object is blurred (high-spatial-frequency information being attenuated) the more the occluding object appears to stand out in apparent depth in front of it. Other related studies have found spatial frequency influences on figure-ground perception (Klymenko \& Weisstein, 1986; Wong \& Weisstein, 1985). Alternate regions of figure-ground reversible configurations were filled with sinusoidal gratings of differing spatial frequency. Whether the configuration was Rubin's (1921/1958) faces/vase (Klymenko \& Weisstein, 1986), a maltese cross (Klymenko \& Weisstein, 1986), a bipartite field (Klymenko \& Weisstein, 1986), or a diskannulus (Wong \& Weisstein, 1985), the regions containing the relatively higher spatial frequency appeared as figure a greater percentage of the time than did the regions

This research was supported by Air Force Office of Scientific Research Grant AFOSR 840115. We thank Victor Klymenko for programming assistance, Mary C. Williams for helpful comments, and James G. May for allowing us to use his image processor to create the demonstration figures. Requests for reprints should be directed to the first author at Department of Otorhinolaryngology and Biocommunication, Kresge Hearing Research Laboratory of the South, Louisiana State University Medical Center, 2020 Gravier Street, Suite A, New Orleans, LA 70112-2234. containing the relatively lower spatial frequency. The larger the octave separation in spatial frequency, the more likely the higher frequency filled regions were organized as figure. While these studies did not directly address the perceived depth created within their stimuli, the implicit depth relations accompanying a figure-ground segregation specified the figure regions (and thus the relatively higher spatial frequency filled regions) closer in depth a greater percentage of the time.

Most pertinent to our present findings are studies that directly examined the effect of spatial frequency on the perception of depth (Frisby \& Mayhew, 1978; Schor \& Howarth, 1986). Frisby and Mayhew (1978) used stimuli consisting of two-dimensional, spatial frequency filtered, rivalrous-textured, random-dot stereograms. They found that a central square centered at 10 cycles per degree (cpd) appeared closer in depth than its surround centered at 2.5 cpd, despite contrary stereo information. Two factors may have contributed to their results. Having a smaller region surrounded by a larger region may have biased the central region to be seen as figure, or "in front," more than as ground, or "behind." Rubin (1921/1958) called this the principle of surroundedness. The second factor may have been the creation of apparent depth by adjacent regions of differing spatial frequency. Schor and Howarth (1986) had subjects stereoscopically align spatial frequency filtered vertical bars with a zero-disparity standard line (with high spatial frequencies present). They found a perceived-depth bias, with subjects placing low spatial frequency filtered bars (less than $1.2 \mathrm{cpd}$ ) stereoscopically closer than the standard.

Our objective was to examine the apparent depth effect due to spatial frequency differences. More specifically, we examined the interaction of depth information due to spatial frequency differences and depth information due to binocular disparity. Observers made judgments about the relative depth of adjacent spatial frequency filled regions. In the first experiment, the binocular disparity that specified the relative depth of the regions was fixed, and observers chose which of the spatial frequency filled regions appeared closer in depth. In Experiments 2-4, observers adjusted the relative disparity between the regions 


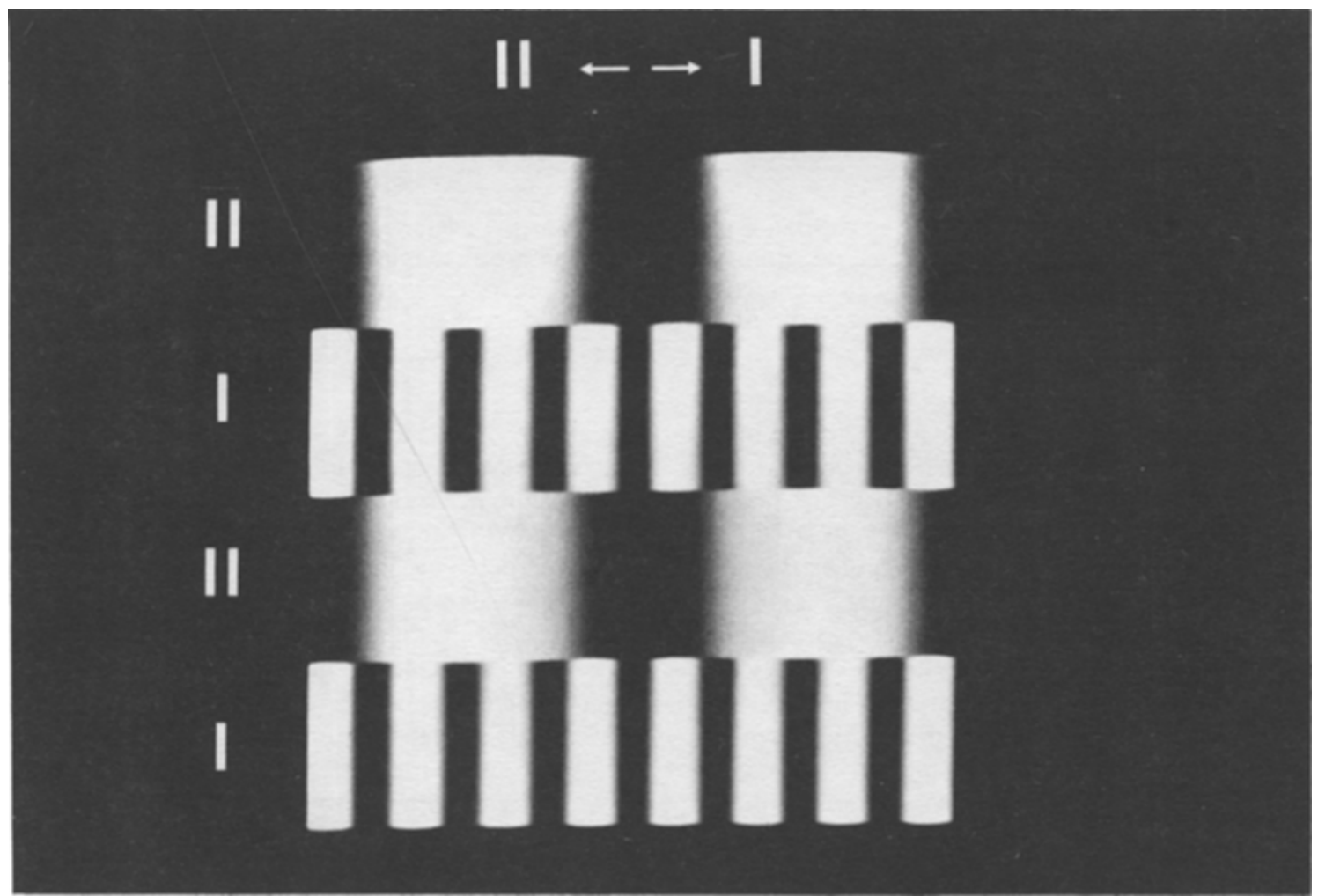

Figure 1. Example of a stimulus used in Experiments 1 and 2. Vertical sine-wave gratings filled the regions labeled I and II. Binocular disparity was created within the regions using the red-green anaglyph method. Subjects made judgments regarding the perceived depth between the regions labeled I and II.

until they appeared to be in the same depth plane. These experiments allowed us to assess the influence of spatial frequency information on perceived depth, using binocular disparity as a measure.

\section{EXPERIMENT 1}

\section{Method}

Subjects. All subjects were undergraduates completing requirements for an introductory psychology course. All had normal or corrected-to-normal vision.

Apparatus and Stimuli. Stimuli were created on a SONY Trinitron color television using an LSI-11/23 computer and a Grinnell Image Processing system. The computer controlled stimulus presentation and data collection.

The configuration used in all experiments is shown in Figure 1. Subjects made depth judgments about the pair of horizontal rectangular regions labeled I relative to the pair labeled II. These alternate regions were filled with sinusoidal gratings (square-wave gratings in Experiment 4) of differing spatial frequency. The frequency filled regions subtended a total of $3^{\circ}$ (width) $\times 4^{\circ}$ (height) visual angle (VA) at a viewing distance of $3.63 \mathrm{~m}$.

Gratings were presented at $90 \%$ contrast with a mean luminance of $21.56 \mathrm{~cd} / \mathrm{m}^{2}$. Three spatial frequencies, $0.5,2.0$, and $5.0 \mathrm{cpd}$, were used. This resulted in frequency differences of 1.32, 2.0, and 3.32 octaves. The three octave conditions were presented under nine disparity conditions for a total of 27 trials. Binocular disparity was created within the different spatial frequency filled regions using the red-green anaglyph method. The gratings were created once in green and again in red. The amount of horizontal shift between the red and green images accounted for the disparity difference between the two eyes. The area surrounding the horizontal rectangular regions was dark, but noticeably lighter than the dark grating bars. This created a window-like effect, with the Is and IIs appearing relatively closer or farther away with respect to each other as seen through the zero-disparity surround. The subjects viewed the stimuli through red/green filters from a chinrest.

Zero disparity and two crossed disparity values (2.4' and 4.2') were combined to create the nine disparity conditions. The disparity differences resulting from these combinations were $1.8^{\prime}, 2.4^{\prime}$, and 4.2 '. These disparity differences created calculated depth differences of $11.3,15.2$, and $27.5 \mathrm{~cm}$, respectively (see Cormack \& Fox, 1985, for formula) ${ }^{1}$ Of the nine disparity conditions, three had no disparity differences between the lower $(\mathrm{LO})$ and higher $(\mathrm{Hi})$ spatial frequency filled regions $\left(\mathrm{Lo}=0^{\prime} / \mathrm{Hi}=0^{\prime} ; \mathrm{Lo}=2.4^{\prime} / \mathrm{Hi}=2.4^{\prime}\right.$; $\mathrm{Lo}=4.2^{\prime} / \mathrm{Hi}=4.2^{\prime}$ ), three had less disparity in the lower frequency regions $\left(\mathrm{Lo}=2.4^{\prime} / \mathrm{Hi}=4.2^{\prime} ; \mathrm{Lo}=0^{\prime} / \mathrm{Hi}=2.4^{\prime} ; \mathrm{Lo}=0^{\prime} / \mathrm{Hi}=4.2^{\prime}\right)$, and three had more disparity in the lower frequency regions $\left(L o=4.2^{\prime} / \mathrm{Hi}=2.4^{\prime} ; \quad L o=2.4^{\prime} / \mathrm{Hi}=0^{\prime} ; \quad L O=4.2^{\prime} / \mathrm{Hi}=0^{\prime}\right)$. These conditions allowed us to compare the influence of spatial frequency and disparity information on the organization of the regions in depth.

Procedure. Three random-dot stereograms (Julesz, 1971) and six single-frequency test stimuli were viewed before the experiment. The single-frequency stimuli contained the same spatial frequency in all regions. The gratings in the Is were $90^{\circ}$ out-of-phase with the gratings in the IIs so the Is and IIs were distinguishable from each other without disparity information (see Figure 2).

Each spatial frequency was presented twice, once with the Is $4.2^{\prime}$ closer and once with the Ils $4.2^{\prime}$ closer. Presentation order for single- 


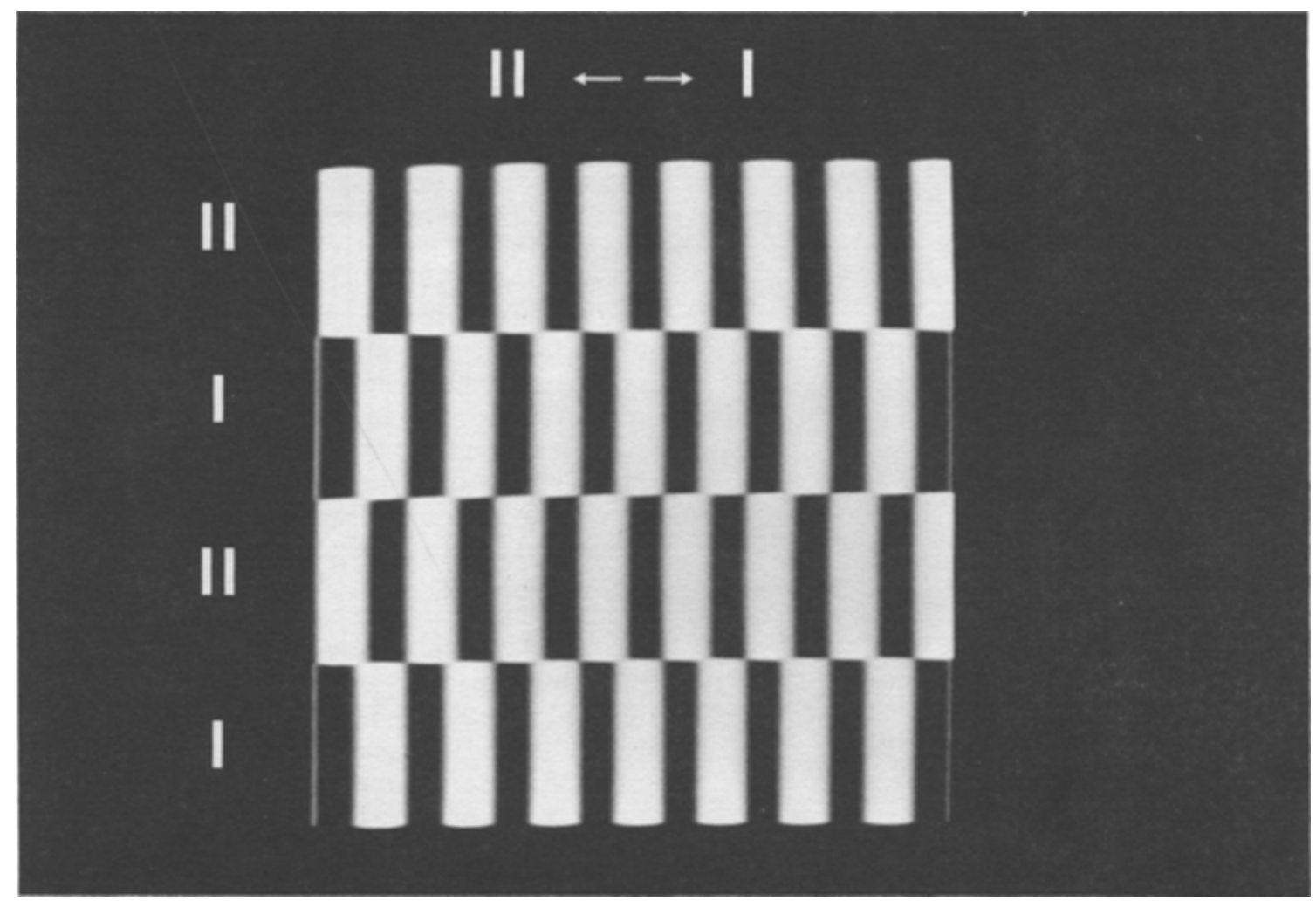

Figure 2. Example of a single-frequency stimulus.

frequency stimuli was random. Only subjects who correctly chose the shape and position in depth of all three stereograms and correctly chose the closer pair of regions in five of the six singlefrequency stimuli participated in the experiment.

Experimental trials were randomized. A stimulus appeared on the screen for a total of $20 \mathrm{sec}$ each trial. After the stimulus first appeared, the subject simply examined the stimulus for $5 \mathrm{sec}$. A tone then signaled the subject to begin responding. During a 15sec response period, the subject indicated whether the regions labeled I or the regions labeled II appeared to be closer in depth. The subject responded by holding a joystick in one position for Is and in another position for IIs. The subject could change his/her choice, but was required to respond as soon as the tone sounded, and to continue responding until the screen was automatically blanked at the end of the $15-\mathrm{sec}$ response period.

\section{Results and Discussion}

The percentage of total viewing time in which the relatively higher spatial frequency regions were perceived as being closer in depth was calculated for each combination of disparity difference and octave separation (see Figure 3). A two-way repeated measures analysis of variance (ANOVA) on these values revealed a significant main effect of octave separation in spatial frequency between the regions $[F(2,22)=22.16, p<.01]$. The main effect of disparity difference and all interactions were not significant. The results indicate that when the difference in spatial frequency between the alternate regions was two or more octaves, the regions containing the relatively higher spatial frequency were perceived closer in depth a significantly greater percentage of the total viewing time, regardless of the disparity condition. As shown in Figure 3 (solid line), even when disparity specified the relatively lower frequency filled regions closer in depth, with a frequency difference of two or more octaves, the higher frequency filled regions were still seen as closer in depth a greater percentage of the time. The data in Figure 3 also indicate that the displays were multistable, particularly for the 1.32-octave stimuli. In the 2.0- and 3.32-octave conditions, the higher spatial frequency regions were seen as being in front the majority of the total viewing time, whereas the lower frequency regions were seen as being in front between $12 \%$ and $19 \%$ of the time. In the 1.32-octave conditions, the lower frequency regions were seen as being in front $57 \%-65 \%$ of the time, indicating that subjects' judgments were less stable, that is, more likely to reverse between low- and highfrequency regions seen as being in front.

In the no-disparity-difference conditions, we had essentially created a new spatial frequency, figure-ground effect (see Klymenko \& Weisstein, 1986; Wong \& Weisstein, 1985) stimulus. Consistent with the previous findings, our results showed no preference for the lower or the higher frequency filled regions to be seen as being in front (or from a figure-ground perspective, as figure) when the frequency difference was near an octave. Sub- 


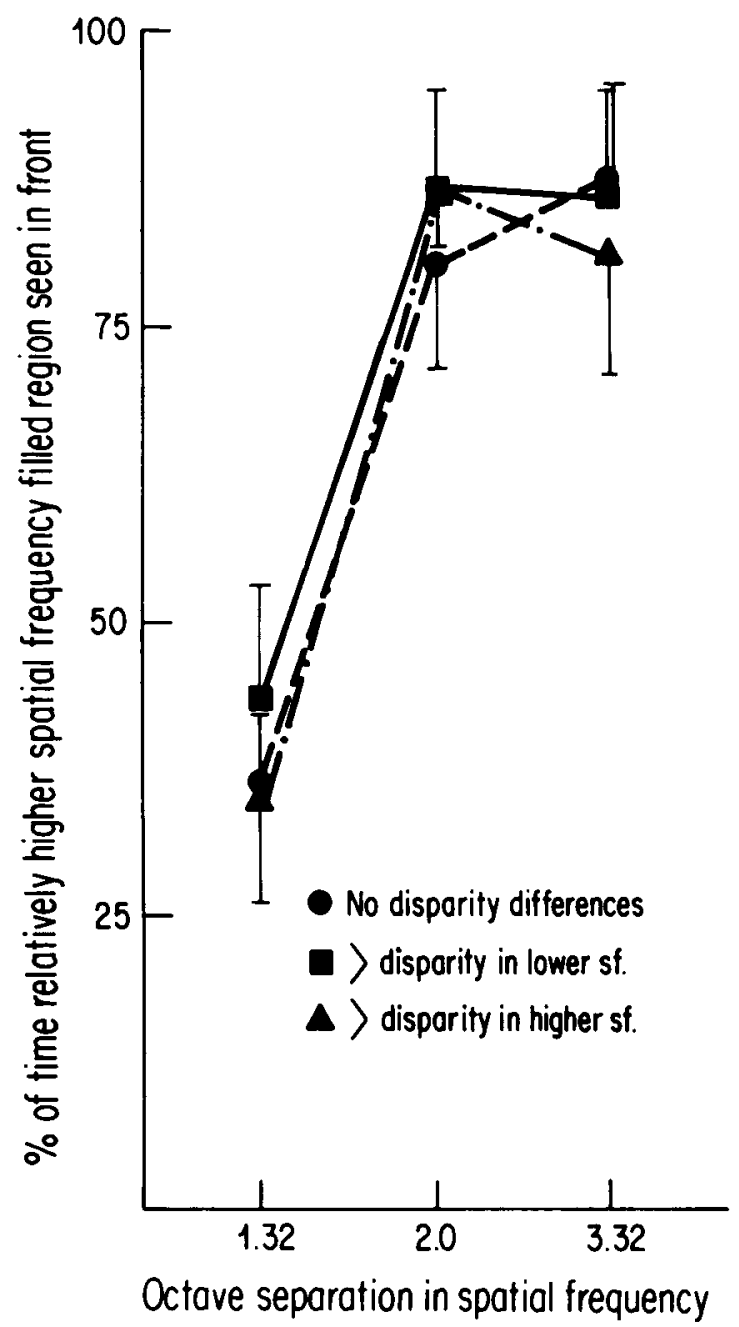

Figure 3. The mean $(N=12)$ percentage of time the relatively higher spatial frequency filled regions were perceived in front of the relatively lower frequency regions in Experiment 1. Means are plotted as a function of octave separation in spatial frequency.

jects apparently had trouble deciding whether the lower or higher frequency regions were closer when the frequency was close; the organization was more stable with 2.0- and 3.32-octave separations.

The stereograms in Figures 4-6 will help demonstrate this spatial frequency influence on perceived depth. The left and right halves of each figure contain four adjacent horizontal rectangular regions filled with sine-wave gratings. $^{2}$ For those who have difficulty in free-fusing, a stereoscope or prism arrangement may be needed to create a single fused image from the two halves of each figure. The white markers at the top and bottom should assist in fusing. The same labeling convention (Is and IIs) will be used. The question to ask when viewing each stereo figure is, Which pair of regions (Is or IIs) appears in front, closer in depth? Figure 6 is an example of a spatial frequency influence on perceived depth, and Figures 4 and 5 illustrate important points concerning the stimu- lus and the interaction of spatial frequency and stereo information. Figure 4 shows that there is no difficulty fusing the low spatial frequency filled regions, or seeing them stereoscopically closer in depth. The organization of the Is as in front should be very stable. Looking at either half of Figure 5 alone, the higher frequency filled IIs might appear as figure in front of the lower frequency filled Is. This would be an example of the spatial frequency figureground effect (Klymenko \& Weisstein, 1986; Wong \& Weisstein, 1985). When the figure is fused as a stereogram however, there is no doubt that the Ils are in front of the Is. Binocular information contributes to a stable organization. Now view Figure 6, again asking which regions appear in front. The organization may seem unstable and tend to reverse with the Is appearing in front, then the IIs, and so forth. The reversibility, and instability of the organization demonstrates the spatial frequency influence on perceived depth. The only difference between Figure 4 and Figure 6 is that a higher spatial frequency grating fills the IIs; the amount of crossed disparity in the Is is the same. If the amount of crossed disparity in the lower frequency filled regions in Figure 6 were reduced, the reversibility of the organization would also be reduced and the higher spatial frequency regions would begin to appear in front more often.

The results of Experiment 1 and Figure 6 indicate that under certain conditions stereo information is abandoned or ignored in favor of spatial frequency information when observers are forced to decide about the relative depth of regions. Would the same trend of results be found when subjects were not forced to make a choice? A method of adjustment was used in the second experiment to address this question.

\section{EXPERIMENT 2}

\section{Method}

Subjects. A new group of introductory psychology students with normal or corrected-to-normal vision participated in this experiment.

Apparatus and Stimuli. The apparatus, stimulus configuration, luminance, and contrast were the same as in Experiment 1 . The horizontal regions subtended a total of $3.3^{\circ}$ (width) $\times 3.6^{\circ}$ (height) VA from a viewing distance of $2.98 \mathrm{~m}$. The spatial frequencies used were $.56,2.26$, and $5.65 \mathrm{cpd}$, resulting in frequency differences of $1.32,2.01$, and 3.33 octaves. The relative disparity between the regions labeled I and II was controlled by the subject. The direction in which a joystick was moved controlled whether the Is or the IIs moved closer, or farther away, in relative disparity steps of $40^{\prime \prime}$ of VA. Moving the joystick to the left brought the IIs closer; moving it to the right brought the is closer. The maximum possible relative disparity between the regions was $6^{\prime} 40^{\prime \prime}$ of VA, which corresponds to a perceived depth of $300.8 \mathrm{~cm}$. This disparity difference consisted of $3^{\prime} 20^{\prime \prime}\left(5 \times 40^{\prime \prime}\right)$ crossed and $3^{\prime} 20^{\prime \prime}\left(5 \times 40^{\prime \prime}\right)$ uncrossed disparity. For example, when moving the Is closer, first $40^{\prime \prime}$ crossed disparity was created in the Is, then $40^{\prime \prime}$ uncrossed disparity was created in the IIs, then another $40^{\prime \prime}$ crossed in the Is, and so forth.

Procedure. A subject was first required to correctly choose three of three random-dot anaglyphs as previously described. Each of the three frequencies was then shown twice as single-frequency stimuli. A sequence of three steps was followed for the first presen- 


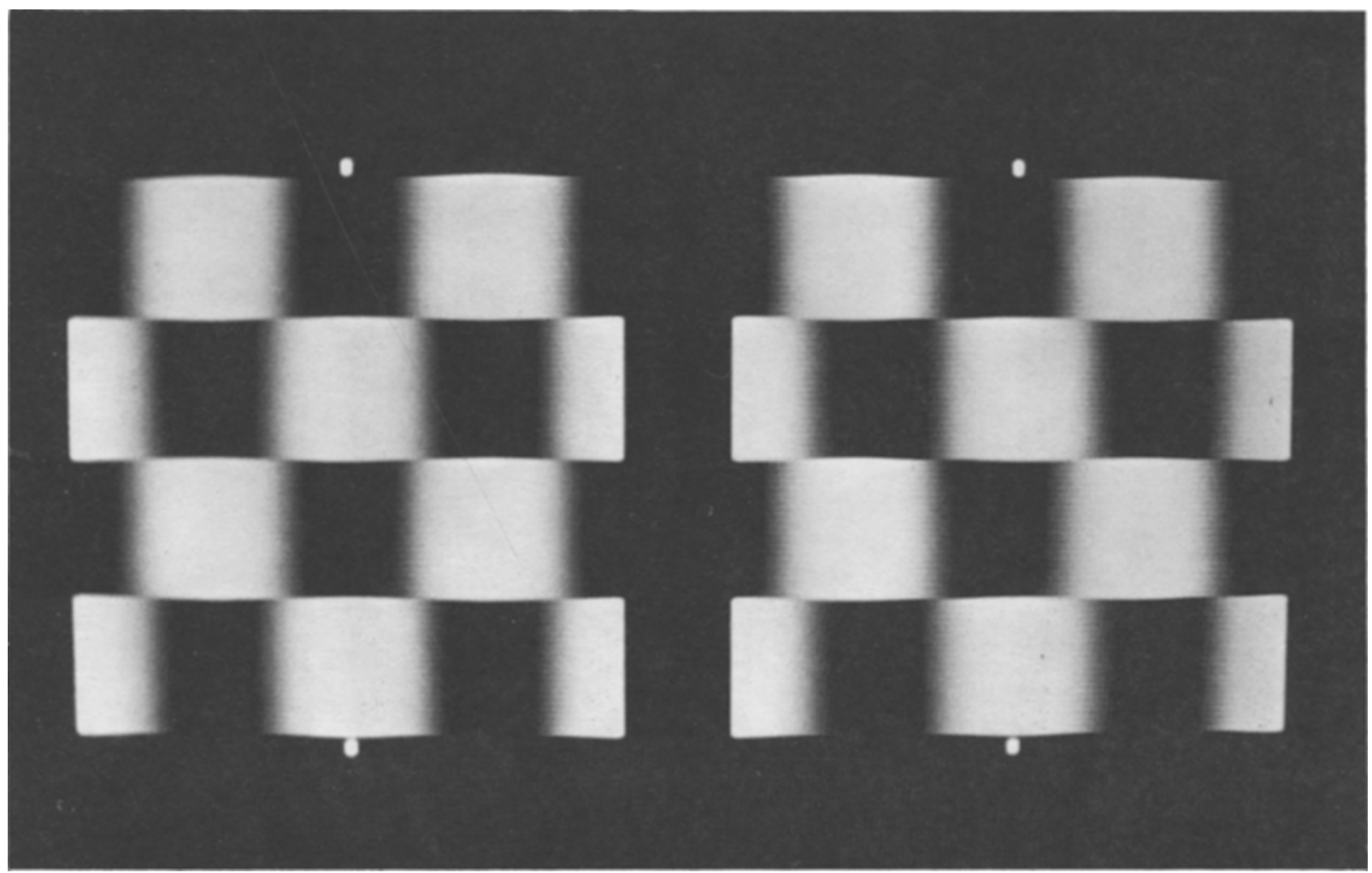

Figure 4. Stereogram of a single-frequency stimulus with crosed disparity desigmeting the first and third horizontal regions choser in depth.

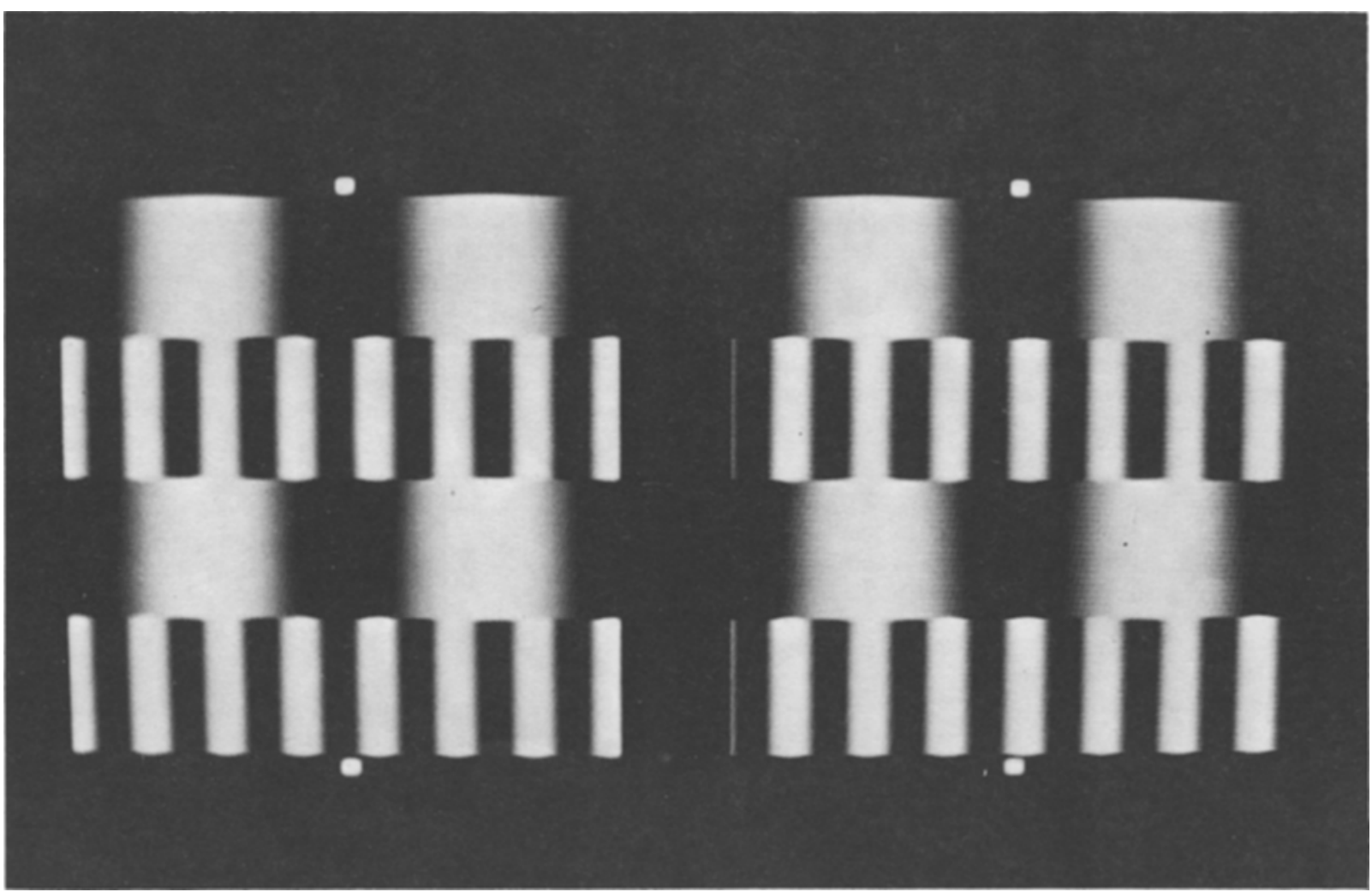

Figure 5. Stereogram with crossed disparity designating the higher spatial frequency filled regions closer in depth. 


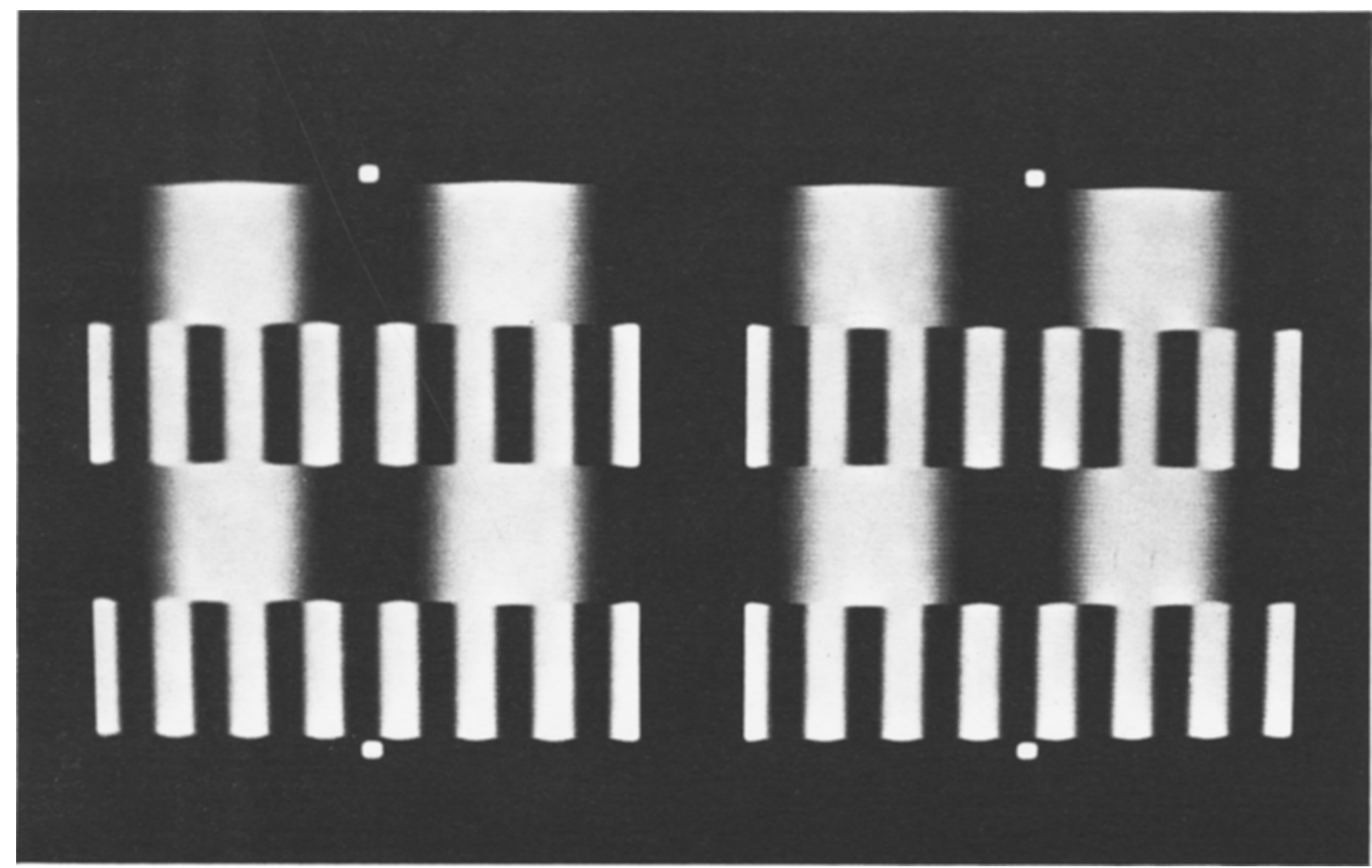

Figure 6. Stereogram demonstrating a spatial frequency depth effect on perceived depth. While crossed disparity is designating the lower spatial frequency filled regions as being closer in depth, the higher frequency filled regions may also appear in front sometimes.

tation of a single-frequency stimulus. Initially the disparity was set to where neither the Is nor the IIs were in front of the other (the neutral position or a zero-disparity difference). The relative movement of the regions in depth was then demonstrated. Next, the disparity difference between the regions was arbitrarily set and subjects chose which regions were closer in depth. This forced-choice procedure was done twice with feedback. During the third step, subjects were instructed on how to adjust the perceived depth between the Is and Ils using the joystick. Setting the disparity to the neutral position was practiced with feedback, until the subject could set it within two steps of zero. The second time a particular singlefrequency stimulus was presented, the subject set the disparity to the neutral position without feedback. Subjects who were unable to perform the task were dropped from the experiment. Sixteen of 18 subjects passed the training session.

During the experiment, the subjects adjusted the regions in depth until they appeared to be in the same depth plane. Each of the three octave conditions was tested four times, twice with the higher frequency in the Is and twice in the IIs. Three single-frequency (i.e., zero octave) catch trials were tested twice. The single-frequency catch trials eliminated subjects who had apparent trouble during the experiment using the stereo information available. Passing criterion for catch trials was a mean disparity setting within two steps of the neutral position. The mean disparity difference for observers passing catch trial criterion was $31.6^{n}$ (less than one step) with a standard error $(S E)$ of $4.4^{\prime \prime}$, thus demonstrating that they were stereo sensitive and able to perform the task quite well. Five of 16 subjects were rejected on this criterion.

\section{Results and Discussion}

The mean disparity settings where the low and high spatial frequency filled regions were perceived in the same depth plane are shown in Table $1 .^{3}$ The spatial frequency differences in octaves are shown on the left. The mean disparity differences between the regions when they were perceived coplanar are shown on the right. Again, the main effect of octave separation in spatial frequency was significant $[F(2,20)=3.67, p<.05]$. According to disparity information, subjects consistently placed the relatively lower frequency regions closer than the higher frequency regions when aligning them in the same perceived depth plane. The disparity setting for the 2.01-octave condition was significantly different from the setting for the 1.32- and 3.33-octave conditions. The difference between the 3.33- and 1.32-octave conditions did not quite reach significance. As in Experiment 1, spatial frequency information appears to strongly influence perceived depth. This influence could actually be canceled by stereo information that specified the opposite depth organization.

\section{EXPERIMENT 3}

In Experiments 1 and 2, the grating stripes within the Is and in the Ils were always in-phase, whereas the gratings between the Is and IIs were always out-of-phase. When either the l's or II's were perceived closer in depth, the regions seen farther away could sometimes be seen as a single continuous surface behind the closer, occluding regions (as some subjects reported). It is common to experience the background as continuing behind figure 
Table 1

Mean and Standard Errors of Disparity Settings (in Seconds of Visual Angle) from Experiments 2-4, When the Lower and Higher Spatial Frequency Regions Appeared in the Same Depth Plane

\begin{tabular}{|c|c|c|c|c|c|c|c|}
\hline \multirow[b]{3}{*}{ Experiment } & \multirow{3}{*}{$\begin{array}{l}\text { Phase Relation } \\
\text { Within Is and IIs }\end{array}$} & \multicolumn{6}{|c|}{ Octave Separation in Spatial Frequency } \\
\hline & & \multicolumn{2}{|c|}{1.32} & \multicolumn{2}{|c|}{2.01} & \multicolumn{2}{|c|}{3.33} \\
\hline & & $M$ & $S E$ & $M$ & $S E$ & $M$ & $S E$ \\
\hline 2 & IN & 5.4 & 28 & 96.4 & 29 & 49.2 & 30 \\
\hline 3 & $\begin{array}{c}\text { IN } \\
\text { OUT }\end{array}$ & $\begin{array}{r}-10.0 \\
1.2\end{array}$ & $\begin{array}{l}14 \\
12\end{array}$ & $\begin{array}{l}67.2 \\
56.0\end{array}$ & $\begin{array}{l}21 \\
26\end{array}$ & $\begin{array}{l}47.2 \\
69.2\end{array}$ & $\begin{array}{l}22 \\
23\end{array}$ \\
\hline 4 & $\begin{array}{l}\text { IN } \\
\text { OUT }\end{array}$ & $\begin{array}{r}-18.0 \\
-4.4\end{array}$ & $\begin{array}{l}9 \\
5\end{array}$ & $\begin{array}{l}-6.4 \\
-2.8\end{array}$ & $\begin{array}{r}11 \\
6\end{array}$ & $\begin{array}{r}-6.4 \\
-22.8 \\
\end{array}$ & $\begin{array}{l}8 \\
8\end{array}$ \\
\hline
\end{tabular}

Note-See text for explanation of experimental conditions. Positive settings indicate the relatively lower frequency regions were placed closer in stereo depth. Negative settings indicate the relatively higher frequency regions were placed closer in depth.

(Rubin, 1921/1958). The alignment of the grating stripes within the Is and IIs may have facilitated the organization of the regions as connected through the Gestalt principle of good continuation. Experiment 3 eliminated this good-continuation cue by placing all regions out-of-phase with each other on half of the trials. Would the spatial frequency influence on perceived depth still be evident?

\section{Method}

Subjects. Twenty-seven students with normal or corrected-tonormal vision participated for course credit. Twenty-five subjects passed the training as described above, and 10 of these subjects' data were within the catch-trial criterion values previously described.

Stimuli and Apparatus. The spatial frequencies $(0.55,2.01$, and 5.65 cpd), apparatus, and viewing conditions were the same as in Experiment 2. The same stimulus combinations used in Experiment 2 were tested four times each, twice when the grating stripes were in-phase (replicating Experiment 2) and twice when the gratings were out-of-phase. In the out-of-phase combinations, the gratings in all regions (Is and IIs) were out-of-phase.

Procedure. The total of 36 trials were presented in random order using the same disparity adjustment procedure as Experiment 2.

\section{Results and Discussion}

The results are shown in Table 1. The data were analyzed in a two-way ANOVA with repeated measures on both factor $A$ and factor $B$. Factor $A$ was the phase angle of the grating stripes within regions labeled I and II. There were two levels of factor $A$, in-phase or out-of-phase. Factor B, spatial frequency separation in octaves, had three levels-1.32, 2.01, and 3.33-and was the only significant variable; $[F(2,18)=4.45, p<.05]$. After collapsing across factor A, a Newman-Keuls probe of factor B found the 2.01- and 3.33-octave conditions significantly different from the 1.32-octave condition. No difference was found between the 2.01- and 3.33-octave conditions. The influence of spatial frequency on perceived depth was found regardless of whether the sinusoidal gratings were in-phase or out-of-phase. Although the regions perceived farther away in depth may be organized as a continuous surface when the grating stripes are in-phase, the results show that this grouping is not vital to the spatial frequency depth effect being studied.

Although fewer subjects passed the catch trial criterion in this experiment (40\%) than did in Experiment 2 (69\%), it should be made clear that this difference was not due to the task's being more difficult with the out-of-phase stimuli and/or that subjects were less accurate in this experiment. For example, accuracy on catch trials was better in this experiment than in the previous experiment, with a mean setting of 24" (SE 3.2") compared with 31.6". Examining catch-trial data of subjects who failed to pass catch-trial criterion also suggests that the out-ofphase stimuli were not the reason for their failure. If outof-phase gratings were more difficult to place in the same stereo depth plane than in-phase gratings, a higher percentage of failures to reach catch-trial criterion for outof-phase catch trials might be anticipated. However, $72 \%$ of the catch-trial failures occurred with in-phase patterns. The 0.56 -cpd in-phase condition accounted for $41 \%$ of the total failures. The 0.56 -cpd out-of-phase condition accounted for only $14 \%$. These data suggest that the fewer people passing catch-trial criterion in this experiment was probably more due to variability of the subject pool than to the use of out-of-phase stimuli.

This experiment demonstrated that the phase relation of the sine-wave gratings was not a factor in the spatial frequency depth effect being examined. Relatively lower spatial frequency filled regions were again placed stereoscopically closer relative to higher spatial frequency filled regions when being aligned in the same apparent depth plane. One factor that may play a role in this spatial frequency depth effect is the blurriness of the images. The 2.01- and 3.33-octave conditions both had the 0.56-cpd frequency as the lower frequency. The 0.56 -cpd grating was noticeably blurrier than the 2.26- and 5.65-cpd sinewave gratings. Pentland (1985) has shown that the relative blurriness of regions can influence the perceived depth between them. In relation to the stereo system however, a stimulus will appear blurry whether closer or farther away relative to the plane of fixation. ${ }^{4}$ Therefore, relative blurriness should not be exclusively associated with a particular depth position. The final experiment addressed this issue of the influence of relative blurriness on perceived depth.

\section{EXPERIMENT 4}

Blur may have influenced the perceived depth relationships between regions in the previous experiments, but 
the superior performance for in-phase 0.56-cpd catch trials in Experiment 3 makes it unlikely that blur hindered the ability to fuse the stereo half-images. This experiment used square-wave gratings to eliminate any influence of blur. Unfortunately, the abrupt edges of square-wave stimuli introduced many high spatial frequency components, in addition to the lower fundamental frequency. These high spatial frequency harmonics for the square-wave stimuli make comparisons across the octave conditions less meaningful than they would be if sine-wave gratings were used. However, the same stimulus combinations and procedures were used for comparison with the previous experiments.

\section{Method}

Subjects. Eleven students with normal or corrected-to-normal vision participated for course credit. All subjects passed the training as described above, and all subjects' data were within catchtrial criterion as previously described.

Stimuli and Apparatus. The stimuli were square-wave gratings with fundamental frequencies of $0.56,2.26$, and $5.65 \mathrm{cpd}$. The spatial frequency combinations and phase angle conditions were the same as in Experiment 3.

Procedure. All procedural details were the same as in Experiment 3. The subjects were screened, trained, and then tested. The subjects grasped the stereo-adjustment procedure more easily than the previous subjects had in viewing sine-wave gratings.

\section{Results and Discussion}

The results are shown in Table 1 . The subjects were almost always able to place the Is and IIs in the same stereo depth plane (zero disparity or neutral position), thus demonstrating that neither spatial frequency separation in octaves nor phase angle had an influence. Their interaction was not significant either. The sharp edges of the square-wave gratings apparently made it easy to fuse the stereo half-images and distinguish differences in depth between the regions. As already mentioned, the sharp edges contained spatial frequency components higher than the fundamental frequency, so the lack of an influence of $o c-$ tave separation was not surprising. It is also unlikely that a spatial frequency effect would have been found in Experiment 1 if square-wave gratings had been used there, considering the fact that subjects consistently placed the different frequency regions near the same stereo position (i.e., the neutral point) in order to align them in the same perceived depth plane.

In Experiments 2 and 3, the regions that were perceptually blurriest (the relatively lower frequency filled regions) remained that way regardless of where they were in stereo depth. When they were placed stereoscopically closer, the blurry portions of the visual field were at a relatively crossed disparity position, whereas sharper portions of the visual field (the relatively higher frequency regions) were at a relatively uncrossed disparity position. Even if subjects redirected their eyes to fixate on the relatively lower frequency filled, stereoscopically closer regions, and could have fused them (which was not possible with our stimuli), they would still have remained blurry. This may be why our stimuli are unusual. In the real world, a closer blurry region becomes sharp when we redirect our eyes to converge and focus on it (which also creates a new zero disparity position). In Experiment 2,1 subject observed that in the $0.56-$ cpd catch trials the stereo closer regions somehow seemed clearer, even though they were still blurry. The main conclusions from these results are that sharp stereo half-images allow for better stereo visibility and that they also reduce the influence of spatial frequency differences (at least in terms of the fundamental frequency) on perceived depth.

\section{GENERAL DISCUSSION}

The present findings bear strongly on recent theories of the perceptual role of different spatial and temporal frequency responses in the visual system. Modern psychophysics has shown the existence of multiple channels in the visual system, each tuned to a relatively narrow range of spatial frequencies and a somewhat broader range of temporal frequencies (Campbell \& Robson, 1968; Robson, 1966). A number of studies have shown that the response to low spatial frequencies is faster in both latency (Breitmeyer, 1975; Lupp, Hauske, \& Wolfe, 1976; Vassilev \& Mitov, 1976) and rise time (Breitmeyer \& Gans, 1977; Robson, 1966; VanNess, Koenderink, \& Bouman, 1967; Watanabe, Mori, Nagata, \& Hiwatashi, 1968; Watson \& Nachmias, 1977) than the response to high spatial frequencies. These channels have long been hypothesized to play different roles in perception, with the low spatial frequency, fast channels leading to an initial background image and the high spatial frequency, slow channels providing a slower, more detailed figural scrutiny of an image (Breitmeyer \& Ganz, 1976; Calis \& Leeuwenberg, 1981; Ginsburg, 1982; Julesz, 1978; Wong \& Weisstein, 1982). What is unexpected in light of these theories is that spatial frequency responses could actually yield a perception of apparent depth, especially an apparent depth that is stereoscopically measurable.

Situations in which stereo information has been dominated by organizational influences have been reported before. For example, when Wheatstone (1838) presented the stereo slides of a landscape pseudoscopically, that is, with the disparity information reversed, the perceived depth was dictated by the formal relationships of the figures rather than by the disparity information. Yellot and Kaiwi (1979) found that observers reported the proper depth in a random-dot stereogram superimposed on a stereo-specified concave face, but they still reported a normal protruding face, not the stereo-specified concave face. It has been suggested that cognitive factors such as knowledge of, and experience with, the normal depth relationships of faces and landscapes lead to the rejection of conflicting stereo information in these unusual situations. However, it seems unlikely that cognitive factors could account for the fact that regions filled with a relatively higher spatial frequency sine-wave grating appear closer in depth than regions filled with a lower frequency grating, especially when stereo information designates the 
lower frequency filled regions as being closer. Observer experience with our stimuli was certainly limited, and if the relative size or width of the grating stripes had been used as a depth cue, it would have cued the lower frequency regions as being closer.

Our results support theories linking the spatial frequency response of the visual system to the perceptual organization of an image. For example, Ginsburg (1982) suggested a relationship between the spatial frequency content of an image and the perceptual information available. Low spatial frequencies transmit basic information about the existence of something in the image and its general form. Higher frequency information would allow for identification and detailed scrutiny. Organizational influences on the spatial frequency response of the visual system also support this view. The detection of high spatial frequency targets has been found to be enhanced in perceived figure relative to perceived ground regions, whereas for low spatial frequency targets, the results have been the reverse (Wong \& Weisstein, 1983). Detection of contour discontinuity, a task requiring detailed (high spatial frequency) information, has also been found to be better when the contour is part of a figure than when it is part of a ground (Weitzman, 1963).

The results of the present experiments and of those showing a spatial frequency influence on figure-ground (Klymenko \& Weisstein, 1986; Wong \& Weisstein, 1985) and depth (Frisby \& Mayhew, 1978; Pentland, 1985) perception support the idea that different spatial frequency responses perform different perceptual functions. A modification to the theories suggested by these results is to specify that relatively high and low spatial frequency responses-that is, spatial frequency responses that are high or low relative to what else is in the image-can generate depth and figure-ground perception. Relatively low spatial frequencies produce a background response, whereas relatively high spatial frequencies produce a figure response that is perceived as being well in front of the background.

The possible contribution of monocular depth cues to the spatial frequency depth effect should also be considered. Two cues, relative size and detail perspective, are closely related to the relative amount of blur between different portions of an image, and this may have influenced the three-dimensional organization of our displays. In the real world, these two cues usually coincide with each other, indicating similar directions of relative depth. For example, when an object is farther away in depth, it will appear smaller and less detail will be evident than when it is closer in depth. Our sine-wave stimuli were somewhat unusual, therefore, because these two cues did not coincide. Relative size may have indicated the wider (larger) bars of the lower frequency gratings to be closer in depth than the narrower (smaller) bars of the higher frequency regions; detail perspective would have indicated the less blurry (more detailed) higher frequency bars to be closer in depth than the blurrier, lower frequency bars.

For our stimuli, the situation becomes even more unusual when we consider the relationship between these two cues, binocular disparity and eye position. If a blurry object behind the plane of fixation is fixated (i.e., the eyes diverge to a more uncrossed eye position), at some point the newly fixated object will remain blurry because it will be at the limits of spatial resolution. This relationship between detail perspective, stereo, and eye position is different when an object closer than the original plane of fixation is refixated. A blurry object closer than the plane of fixation will be focused when the eyes fixate it (move to a more crossed eye position). This is possible to within inches of the eyes. Unlike the real world, however, if the stereo closer regions in our sine-wave stimuli could have been fixated and fused, they would have remained blurry. Detail perspective may therefore have had some influence on seeing the relatively higher frequency regions as being closer in depth, particularly at spatial frequency differences equal to or greater than two.

Relative blur has been used successfully by film producers to create an impression of depth and to influence where a viewer should look in a scene. For example, the out-of-focus back of a person partially occluding a sharply focused person accentuates the focused person farther away, drawing our attention to that person. While the film producer creates a particular depth relationship by using relative blur, relative size, and interposition, in our images there was no obvious reason to perceive the higher frequency regions as occluding the lower frequency regions or vice versa. If interposition had influenced the results, it would have been evident in Experiment 3 where good continuation was evaluated by placing regions of the same spatial frequency in-phase or out-of-phase. A region that continues behind another is usually occluded by the region closer in depth. Because good continuation did not have a significant influence on the perceived depth relationships in Experiment 3, it seems unlikely that interposition had a significant influence either. A more effective way of evaluating the contribution of relative size and interposition cues on the spatial frequency depth effect might be to vary the area of the regions containing the gratings. The present results show that, at least for regions of equal area, spatial frequency relationships between the different regions of our images strongly contributed to the perceived depth within them.

In conclusion, the experiments reported show a spatial frequency depth effect on perceived depth. Regions filled with relatively higher spatial frequency sine-wave gratings appeared closer in depth than regions filled with relatively lower spatial frequency sine-wave gratings, even when stereo information designated the opposite depth relationship. Lower frequency regions had to be placed closer in depth relative to the higher frequency regions in order to localize them in the same perceived depth 
plane. The relationship of depth perception to spatial frequency may be so fundamental that once regions are organized in depth according to spatial frequency relationships, their representation may prevail despite contrary stereo depth information.

\section{REFERENCES}

Breitmeyer, B. (1975). Simple reaction time as a measure of the temporal response properties of transient and sustained channels. Vision Research, 15, 1411-1412.

BreItMEYER, B., \& GANZ, L. (1976). Implications of transient and sustained channels for theories of visual pattern masking, saccadic suppression, and information processing. Vision Research, 83, 1-36.

Breitmeyer, B., \& GANZ, L. (1977). Temporal studies with flashed gratings: Inferences about human transient and sustained channels. Vision Research, 17, 861-865.

Brown, J. M., Weisstein, N. (1985). Spatial frequency influences on the perception of depth. Optics News, 11, 99.

Calus, G., Leeuwenberg, J. (1981). Grounding the figure. Journal of Experimental Psychology, 7, 1386-1397.

CampBell, F. W., RoBson, J. G. (1968). Application of Fourier analysis to the visibility of gratings. Journal of Physiology, 197, 551-566.

Cormack, R., Fox, R. (1985). The computation of disparity and depth in stereograms. Perception \& Psychophysics, 38, 375-380.

Frisby, J. P., \& MAYHEW, J. E. W. (1978). Depth inversion in randomdot stereograms. Perception, 7, 661-678.

GinsBURG, A. P. (1982). On the filter approach to understanding the perception of visual form. In D. G. Albrecht (Ed.), Recognition of pattern and form (pp. 215-262). Hillsdale, NJ: Erlbaum.

Julesz, B. (1971). Foundations of cyclopean perception. Chicago: University of Chicago Press.

JuLesz, B. (1978). Perceptual limits of texture discrimination and their implications to figure-ground separation. In E. Leeuwenberg (Ed.), Formal theories of perception (pp. 205-216). New York: Wiley.

KaufMaN, L. (1974). Sight and mind. New York: Oxford University Press.

KLymenko, V., \& Weisstein, N. (1986). Spatial frequency differences can determine figure-ground organization. Joumal of Experimental Psychology: Human Perception \& Performance, 12, 324-330.

LuPP, U., Hauske, G., \& Wolfe, W. (1976). Perceptual latencies to sinusoidal gratings. Vision Research, 16, 969-972.

Pentland, A. P. (1985). The focal gradient: Optics ecologically salient. Investigative Ophthalmology \& Visual Science, 26, 243. (Abstract)

RoBson, J. G. (1966). Spatial and temporal contrast sensitivity functions of the visual system. Joumal of the Optical Society of America, 56, 1441-1442.

Rubin, E. (1958). Figure and ground. In D. C. Beardslee \& M. Wertheimer (Eds.), Readings in perception (pp. 194-203). Princeton, NJ: Van Norstrand. (Original work published in 1921)

SCHOR, C. M., HowARTH, P. A. (1986). Suprathreshold stereo-depth matches as a function of contrast and spatial frequency. Perception, 15, 249-258.

VAn Ness, F. L., Kornderink, J. J., \& Bouman, M. A. (1967). Spatiotemporal modulation transfer in the human eye. Journal of the Optical Society of America, 57, 1082-1087.

Vassilev, A., Mitov, D. (1976). Perception time and spatial frequency. Vision Research, 16, 89-92.

Watanabe, A., More, T., Nagata, S., \& Hiwatashl, K. (1968). Spatial sine-wave responses of the human visual system. Journal of the Optical Society of America, 8, 1245-1263.

Watson, A. B., Nachmias, J. (1977). Patterns of temporal interac- tion in the detection of gratings. Journal of the Optical Society of America, 17, 893-902.

Weitzman, B. (1963). A threshold difference produced by a figureground dichotomy. Journal of Experimental Psychology, 66, 201-205.

Whentstone, C. (1838). Contributions to the physiology of visionPart the first: On some remarkable and hitherto unobserved phenomena of binocular vision. Philosophical Transactions, Royal Society (London), 371-394

Wong, E., Weisstein, N. (1982). A new perceptual contextsuperiority effect: Line segments are more visible against a figure than against a ground. Science, 218, 587-589.

WONG, E., WeISSTEIN, N. (1983). Sharp targets are detected better against a figure, and blurred targets are detected better against a ground. Joumal of Experimental Psychology: Human Perception \& Performance, 9, 194-202.

WONG, E., WeISSTEIN, N. (1985). Spatial frequency affects figureground organization. Investigative Ophthalmology \& Visual Science, 26, 281. (Abstract)

WoODson, W. E. (1981). Human factors design handbook. New York: McGraw-Hill.

Yellot, J. I., \& KAIWI, J. L. (1979). Depth inversion despite stereopsis: The appearance of random-dot stereograms on surfaces seen in reverse perspective. Perception, 8, 135-142.

\section{NOTES}

1. Some distortions produced in photographing the stimuli from a television may be visible when sighting down the horizontal divisions between the Is and IIs in Figures 1, 2, 4, 5, and 6. These distortions (narrow white bands and/or a curving edge) were not present in the original stimuli. The labels at the top and on the left side of Figures 1 and 2 were actually black on the gray background. For photographic reproduction purposes, they have been made white in these figures.

2. Calculations of depth were based on a median interocular distance distance of $6.32 \mathrm{~cm}$ (Woodson, 1981).

3. Mean settings were first calculated according to where the regions were placed relative to each other in disparity steps (i.e., 40" steps). The mean for a subject at a particular octave condition was based on four settings. For example, if the lower frequency regions were placed closer, they may have had settings of $7,3,5$, and 4 , resulting in a mean of 4.75. The means for all subjects were calculated this way and used in the data analysis. The final means and standard errors for each condition across all subjects were then converted to VA for Table 1.

4. All subjects were instructed not to move their heads in the chinrest and to gaze toward the middle of the TV screen. Some subjects in Experiments 2 and 3 had difficulty in placing the single-frequency stimuli in the same depth plane during initial training. They were advised to look along the edge where the Is and IIs meet, because this is where the regions would shear away from each other in depth. Once they were familiar with the task, it was stressed that they were to look toward the center of the screen during the experiment. Any eye movements by subjects would at most have been $2^{\circ}$ left or right of center (the stimuli were $4^{\circ}$ wide). A fixation point was not initially used because it introduces high spatial frequency components (spatial frequency being one of our variables) and occludes part of the stimulus. After Experiment 3,3 subjects were run with a fixation point present (to insure plane of fixation). The procedure was the same as in Experiment 2 (no out-of-phase conditions, since phase information was shown to have no influence in Experiment 3). Their mean settings for the three octave conditions were $23.2^{\prime \prime}(1.32), 80.0^{\prime \prime}(2.01), 56.8^{\prime \prime}(3.33)$. If there was any difference, these data exhibited a slightly greater spatial frequency influence than did those of either Experiment 2 or Experiment 3.

(Manuscript received September 21, 1987; revision accepted for publication March 2, 1988.) 